\title{
Development of machine learning algorithms for prediction of discharge disposition after elective inpatient surgery for lumbar degenerative disc disorders
}

\author{
Aditya V. Karhade, BE, ${ }^{1}$ Paul Ogink, MD,, Quirina Thio, MD, ${ }^{1}$ Marike Broekman, MD, PhD, JD, ${ }^{2}$ \\ Thomas Cha, MD, MBA, ${ }^{1}$ William B. Gormley, MD, MBA, MPH, ${ }^{3}$ Stuart Hershman, MD, ${ }^{1}$ \\ Wilco C. Peul, MD, PhD, MBA, ${ }^{2}$ Christopher M. Bono, MD, ${ }^{1}$ and Joseph H. Schwab, MD, MS ${ }^{1}$
}

${ }^{1}$ Department of Orthopedic Surgery, Massachusetts General Hospital, Harvard Medical School, Boston, Massachusetts; 2Department of Neurosurgery, Leiden University Medical Center, Leiden, The Netherlands; and 'Department of Neurosurgery, Brigham and Women's Hospital, Harvard Medical School, Boston, Massachusetts

OBJECTIVE If not anticipated and prearranged, hospital stay can be prolonged while the patient awaits placement in a rehabilitation unit or skilled nursing facility following elective spine surgery. Preoperative prediction of the likelihood of postoperative discharge to any setting other than home (i.e., nonroutine discharge) after elective inpatient spine surgery would be helpful in terms of decreasing hospital length of stay. The purpose of this study was to use machine learning algorithms to develop an open-access web application for preoperative prediction of nonroutine discharges in surgery for elective inpatient lumbar degenerative disc disorders.

METHODS The American College of Surgeons National Surgical Quality Improvement Program was queried to identify patients who underwent elective inpatient spine surgery for lumbar disc herniation or lumbar disc degeneration between 2011 and 2016. Four machine learning algorithms were developed to predict nonroutine discharge and the best algorithm was incorporated into an open-access web application.

RESULTS The rate of nonroutine discharge for 26,364 patients who underwent elective inpatient surgery for lumbar degenerative disc disorders was $9.28 \%$. Predictive factors selected by random forest algorithms were age, sex, body mass index, fusion, level, functional status, extent and severity of comorbid disease (American Society of Anesthesiologists classification), diabetes, and preoperative hematocrit level. On evaluation in the testing set $(n=5273)$, the neural network had a c-statistic of 0.823 , calibration slope of 0.935 , calibration intercept of 0.026 , and Brier score of 0.0713 . On decision curve analysis, the algorithm showed greater net benefit for changing management over all threshold probabilities than changing management on the basis of the American Society of Anesthesiologists classification alone or for all patients or for no patients. The model can be found here: https://sorg-apps.shinyapps.io/discdisposition/.

CONCLUSIONS Machine learning algorithms show promising results on internal validation for preoperative prediction of nonroutine discharges. If found to be externally valid, widespread use of these algorithms via the open-access web application by healthcare professionals may help preoperative risk stratification of patients undergoing elective surgery for lumbar degenerative disc disorders.

https://thejns.org/doi/abs/10.3171/2018.8.FOCUS18340

KEYWORDS discharge disposition; machine learning; predictive analytics; spine surgery; value-based care

$\mathrm{V}$ ALUE-BASED care requires elimination of low-value practices such as prolonged length of stay due to the delayed recognition of the need for nonhome discharge. ${ }^{23,24}$ Delays in discharge while awaiting insurance precertification, after being medically cleared, have been demonstrated to be a driver of increased inpatient costs. ${ }^{28}$ This is derived, at least in part, by significant variability in the care of the surgical patient. , $18,26^{2}$

Spine surgery is one of 17 care episodes that account for half of Medicare spending, with lumbar degenerative disc disorder being the most common indication for elective spine surgery. ${ }^{4,29}$ The ability to predict which patients will

ABBREVIATIONS ASA = American Society of Anesthesiologists; $A U C=$ area under the curve; BMI = body mass index; NSQIP = National Surgical Quality Improvement Program. 
require postoperative discharge to an inpatient rehabilitation center or nursing facility, i.e., nonroutine discharge, could help facilitate preoperative insurance precertification for nonroutine discharge.

Previous studies of discharge destination after spine surgery have highlighted several risk factors for nonroutine discharge. , $7,10,17,19-22,27$ Few have developed predictive models, and none have considered model evaluation metrics such as calibration and decision analysis. Furthermore, none have applied modern techniques such as machine learning.

The purposes of this study were as follows: 1) to develop machine learning models for preoperative prediction of nonroutine discharge in patients undergoing elective spine surgery for lumbar disc displacement or disc degeneration in a large multiinstitutional surgical registry and 2) to deploy these models as open-access web applications for healthcare professionals.

\section{Methods \\ Guidelines}

The Transparent Reporting of multivariable Prediction Models for Individual Prognosis or Diagnosis (TRIPOD) and the Guidelines for Developing and Reporting Machine Learning Predictive Models in Biomedical Research were followed. ${ }^{3,14}$ The present study is a retrospective machine learning classification study (outcome was binary categorical) for preoperative prognostication in patients undergoing spine surgery for lumbar degenerative disc disorders.

\section{Data Source}

The National Surgical Quality Improvement Program (NSQIP) is a clinical registry of 30-day outcomes extensively studied in spine surgery. ${ }^{11,25}$ Institutional review board approval specific to this study was not sought as the de-identified NSQIP data have been previously exempted for individual review by our institutional review board.

\section{Participants}

We queried the NSQIP database to identify patients in whom the following inclusion criteria were met: 1) inpatient operation, 2) elective surgery, 3) Current Procedural Terminology code for decompression or decompression and fusion at lumbar levels, 4) primary postoperative diagnosis of the International Classification of Disease for lumbar disc displacement or lumbar disc degeneration (which for convenience we collectively refer to as lumbar degenerative disc disorders), 5) general anesthesia, 6) American Society of Anesthesiologists (ASA) classification I-IV, 7) subspecialty neurosurgery or orthopedics, and 8) year of operation 2011 to 2016.

We excluded patients with the following factors: 1) preoperative wound infection, 2) preoperative systemic inflammatory response syndrome (SIRS), sepsis, or septic shock, 3) emergency surgery, 4) admission from any setting other than home, and 5) ventilator dependent preoperatively. NSQIP procedural codes were reviewed to ensure that patients did not undergo additional major surgical procedures unrelated to the spine surgery.

\section{Outcomes}

The primary dependent variables evaluated was discharge disposition, categorized as a binary outcome (routine, nonroutine). Nonroutine discharge was defined as any discharge other than to home.

\section{Candidate Covariates}

The following preoperative covariates were extracted and considered candidate features for final modeling if missing in fewer than $30 \%$ of patients: age (continuous), sex (male, female), body mass index (BMI) (continuous), fusion (yes, no), approach (anterior, posterior, combined), level of surgery (1 or 2 levels, 3 or more levels), instrumentation (yes, no), preoperative functional status (independent, dependent), preoperative hematocrit level (continuous), preoperative white blood cell count (continuous), platelet count (continuous), preoperative creatinine level (continuous), preoperative sodium level (continuous), preoperative albumin level (continuous), ASA classification (I, II, III, IV), steroid use for chronic condition, diabetes (none, requiring oral medication, insulin dependent), current smoking status, hypertension requiring medication, bleeding disorders, history of congestive heart failure, and chronic obstructive pulmonary disease. Multiple imputation with chained equations was used to impute missing data.

\section{Statistical Analysis}

A stratified 80:20 split of the available data was carried out. The training set was used for algorithm training and assessment of performance by 10 -fold cross-validation. All study variables were entered into random forest algorithms, and recursive feature selection was used to identify the subset of features employed in final modeling. ${ }^{13}$ Neural network, support vector machine, Bayes point machine, and boosted decision tree models were subsequently trained to predict 30 -day mortality. $8,13,16$ The bestperforming model was used to predict discharge disposition in the testing set. Model performance was assessed by discrimination (c-statistic, receiver operating curve), calibration (calibration plot, calibration slope, calibration intercept), and overall performance (Brier score). The null model Brier score (assigning a probability of nonroutine discharge equivalent to the prevalence of nonroutine discharge) was calculated for comparison.

\section{Application Development}

The best algorithm across the model performance metrics for predicting nonroutine discharge disposition was incorporated into an interactive interface and deployed as an open-access web application (Fig. 1). The Anaconda Distribution (Anaconda, Inc.), Microsoft Azure (Microsoft Corporation), R version 3.4.4 (The R Foundation), RStudio version 1.0.153 (RStudio), and Python version 3.6 (Python Software Foundation) were used for data analysis, model creation, and web application development.

\section{Results}

The rate of nonroutine discharge in 26,364 patients undergoing elective inpatient surgery for lumbar degen- 


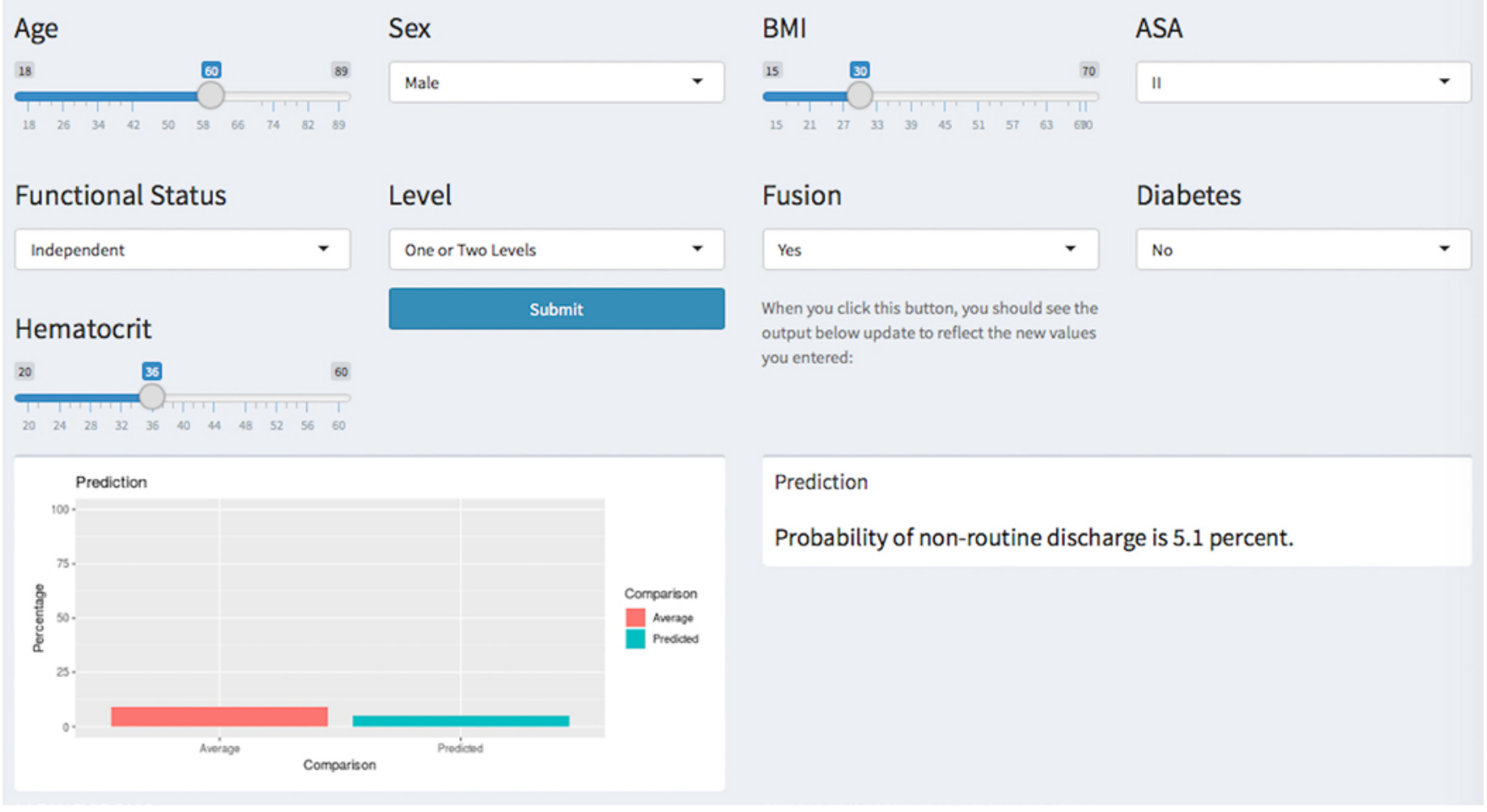

FIG. 1. Open-access web application interface for the final preoperative discharge prediction model.

erative disc disorders was $9.28 \%$. Predictive factors selected by random forest algorithms were age, sex, BMI, fusion, level, functional status, extent and severity of comorbid disease (ASA classification), diabetes, and preoperative hematocrit level. The median age of the cohort was 53 years (interquartile range [IQR] 42-65 years), $46.9 \%$ were female, and the median BMI was $29.6 \mathrm{~kg}$ / $\mathrm{m}^{2}$ (IQR $25.98-33.90 \mathrm{~kg} / \mathrm{m}^{2}$ ). Overall, $56.2 \%$ of patients underwent fusion, $82.5 \%$ underwent surgery via the posterior approach, and $36.7 \%$ had surgery at 3 or more levels (Table 1).

\section{Training Set Performance}

The discrimination of the algorithms on the training set ranged from a c-statistic of 0.773 for the support vector machine to c-statistic of 0.815 for the neural network (Table 2). The calibration slope ranged from 0.684 for the boosted decision tree to 1.015 for the Bayes point machine (Fig. 2). The calibration intercept ranged from -0.026 for the neural network to 0.001 boosted decision tree. On overall performance assessment, the Brier score ranged from 0.0725 for the neural network to 0.0768 for the boosted decision tree. In comparison, the null model Brier score was 0.086 . The neural network was chosen as the final model with superior performance across discrimination, calibration, and Brier score. The model was incorporated into an interactive web application and deployed as an open access platform here: https://sorg-apps.shinyapps. io/discdisposition/.

\section{Testing Set Performance}

On evaluation in the testing set $(n=5273)$, the neural network had a c-statistic of 0.823 (Fig. 3), calibration slope of 0.935, calibration intercept of 0.026, and Brier score of 0.0713 (Fig. 4). On decision curve analysis, the algorithm showed greater net benefit for changing management over all threshold probabilities than changing management on the basis of the ASA classification alone or for all patients or for no patients (Fig. 5).

\section{Discussion}

Four machine learning algorithms were successfully developed for prediction of nonroutine discharge disposition after elective inpatient surgery for lumbar disc herniation or disc degeneration disorders. The best model, the neural network, performed well on internal validation and was subsequently incorporated into an open-access web application for healthcare professionals.

The features selected by random forest algorithms for prediction of nonroutine discharge concurred with previous studies of risk factors for nonroutine discharge. McGirt et al. previously studied 6921 elective lumbar spine surgery patients with a $9.4 \%$ rate of nonroutine discharge from the Quality and Outcomes Database and identified as risk factors age, ASA classification, nonindependent ambulation, Oswestry Disability Index score, diabetes, and fusion procedure. ${ }^{17} \mathrm{Di}$ Capua et al. studied the American College of Surgeons' NSQIP database for risk factors 
TABLE 1. Baseline characteristics of study population of 26,364 patients

\begin{tabular}{|c|c|c|}
\hline Variable & Definition & Value \\
\hline \multirow[t]{2}{*}{ Age (yrs) } & Median (IQR) & $53(42-65)$ \\
\hline & Missing, no. (\%) & $32(0.1)$ \\
\hline \multirow[t]{2}{*}{ Sex } & Female, no. (\%) & $12,356(46.9)$ \\
\hline & Male, no. (\%) & $14,008(53.1)$ \\
\hline \multirow[t]{2}{*}{$\mathrm{BMI}\left(\mathrm{kg} / \mathrm{m}^{2}\right)$} & Median (IQR) & $29.6(26.0-33.9)$ \\
\hline & Missing, no. (\%) & $150(0.36)$ \\
\hline \multirow[t]{3}{*}{ Functional status } & Independent, no. (\%) & $25,859(98.1)$ \\
\hline & Dependent, no. (\%) & $351(1.3)$ \\
\hline & Missing, no. (\%) & $154(0.6)$ \\
\hline Fusion, no. (\%) & & $14,821(56.2)$ \\
\hline $\begin{array}{l}\text { Instrumentation, } \\
\text { no. (\%) }\end{array}$ & & $12,526(47.5)$ \\
\hline \multirow[t]{3}{*}{ Approach } & Posterior, no. (\%) & $21,738(82.5)$ \\
\hline & Anterior, no. (\%) & $2285(8.7)$ \\
\hline & Combined, no. (\%) & $2341(8.9)$ \\
\hline \multirow[t]{2}{*}{ Levels } & 1 or 2 levels, no. (\%) & $16,684(63.3)$ \\
\hline & 3 or more levels, no. (\%) & $9680(36.7)$ \\
\hline \multirow[t]{4}{*}{ ASA class } & I, no. $(\%)$ & $1762(6.7)$ \\
\hline & II, no. (\%) & $14,876(56.4)$ \\
\hline & III, no. (\%) & $9370(35.5)$ \\
\hline & IV, no. (\%) & $356(1.4)$ \\
\hline \multirow[t]{2}{*}{ Hematocrit level } & Median (IQR) & $41.7(39.0-44.4)$ \\
\hline & Missing, no. (\%) & $2389(9.1)$ \\
\hline \multirow{2}{*}{$\begin{array}{l}\text { White blood cell } \\
\text { count }\left(\times 10^{9} / \mathrm{L}\right)\end{array}$} & Median (IQR) & $7.2(5.9-8.7)$ \\
\hline & Missing, no. (\%) & $2687(10.2)$ \\
\hline \multirow{2}{*}{$\begin{array}{l}\text { Platelet count } \\
\qquad\left(\times 10^{9} / \mathrm{L}\right)\end{array}$} & Median (IQR) & $241(203-285)$ \\
\hline & Missing, no. (\%) & $2702(10.2)$ \\
\hline \multirow{2}{*}{$\begin{array}{l}\text { Sodium level } \\
\text { (mEq/L) }\end{array}$} & Median (IQR) & $139(138-141)$ \\
\hline & Missing, no. (\%) & $3681(14.0)$ \\
\hline \multirow{2}{*}{$\begin{array}{l}\text { Creatinine level } \\
\qquad(\mathrm{mg} / \mathrm{dl})\end{array}$} & Median (IQR) & $0.87(0.73-1.00)$ \\
\hline & Missing, no. (\%) & $3864(14.7)$ \\
\hline \multirow[t]{2}{*}{ Albumin (g/dl) } & Median (IQR) & $4.2(3.9-4.5)$ \\
\hline & Missing, no. (\%) & $16,064(60.9)$ \\
\hline \multirow[t]{3}{*}{ Diabetes } & No & $22,501(85.3)$ \\
\hline & Oral medication, no. (\%) & $2624(10.0)$ \\
\hline & Insulin dependent, no. (\%) & $1239(4.7)$ \\
\hline $\begin{array}{l}\text { Hypertension, no. } \\
\text { (\%) }\end{array}$ & & $11,614(44.1)$ \\
\hline $\begin{array}{l}\text { Current smoker, } \\
\text { no. }(\%)\end{array}$ & & $6513(24.7)$ \\
\hline $\begin{array}{l}\text { Chronic obstruc- } \\
\text { tive pulmonary } \\
\text { disease, no. }(\%)\end{array}$ & & $851(3.2)$ \\
\hline Chronic steroid use & & $864(3.3)$ \\
\hline Bleeding disorder & & $303(1.1)$ \\
\hline
\end{tabular}

of nonroutine discharge after anterior cervical discectomy and fusion and reported age, obesity, diabetes, functional status, and ASA classification as predictive factors for discharge to facility other than home. ${ }^{6}$ Kanaan et al. conducted a single-center retrospective analysis of 339 patients undergoing lumbar laminectomy and identified age, distance walked during hospital stay, and length of stay as predictors of discharge to home..$^{10}$ In a retrospective review of 18,122 hospitalizations for lumbar spine procedures, Deyo et al. described age and spinal arthrodesis as risk factors for discharge to a nursing home, postoperative complications, length of hospitalization, and total charges. $^{5}$

In spine surgery, others have previously created risk scores and calculators based on regression analysis for preoperative prediction concerning nonhome discharge. The Carolina-Semmes grading scale created by McGirt et al. for nonhome discharges in elective lumbar spine surgery achieved an area under the curve (AUC) equal to $0.731 .{ }^{17}$ In surgery overall, Hyder et al. studied 88,068 inpatient general and vascular surgery patients with a $13.4 \%$ rate of nonhome discharges and derived a logistic regression-based calculator using age, ASA classification, elective surgery, and preadmission residence. ${ }^{9}$ Validation of the algorithm in 19,514 orthopedic patients with a $61.5 \%$ rate of home discharge resulted in a c-statistic of 0.876 . It is striking to note the discrimination of the overall orthopedic algorithm by Hyder et al. (AUC $=0.876$ ) compared to the elective lumbar spine-specific scale created by McGirt et al. (AUC $=0.731)$. The rates of nonhome discharge in the subsets of patients undergoing elective inpatient surgery for lumbar disc disease in the present study and the study of lumbar spine surgery patients by McGirt et al. were significantly less than that for the overall orthopedic population studied by Hyder et al. In addition, the incorporation of spine-specific variables contrasts with the calculator previously proposed by Hyder et al. for all orthopedic patients.

The work presented here extends prior prediction studies by using machine learning for the most common population of patients undergoing spine surgery. Furthermore, this work has demonstrated discrimination comparable to or greater than existing studies that focused on elective lumbar spine surgery and utilized critical model assessment metrics neglected by prior studies. The calibration analysis presented in the present study shows the drawbacks of focusing on discrimination alone, as the boosted decision tree achieved a c-statistic greater than the support vector machine but performed much worse on calibration.

The decision curve analysis provides practical insight into the potential impact of the model. For example, consider a hypothetical case in which obtaining preoperative insurance precertification was the proposed change in management. If the threshold probability of nonroutine discharge for deciding to obtain preoperative insurance precertification for a particular hospital were $20 \%$, then the relative weight of true positives to false positives would be approximately 1 to 4 and using the model would result in a standardized net benefit of approximately 0.25 . In the testing set, at a threshold of $20 \%$ predicted prob- 
TABLE 2. Model performance on training set, $n=21,091$

\begin{tabular}{lcccc}
\hline Performance Metric & Boosted Decision Tree & Support Vector Machine & Bayes Point Machine & Neural Network \\
\hline C-statistic & 0.802 & 0.773 & 0.814 & 0.815 \\
\hline Calibration slope & 0.684 & 0.984 & 1.015 & 0.896 \\
\hline Calibration intercept & 0.001 & -0.002 & -0.004 & -0.026 \\
\hline Brier score & 0.0768 & 0.0762 & 0.0730 & 0.0725 \\
\hline Null model Brier score & & \multicolumn{2}{c}{0.086} & \\
\hline
\end{tabular}

ability, the positive predictive value for the neural network was $33 \%$ and the sensitivity (true positive rate) was 54\%. At this threshold of $20 \%$ predicted probability, half of all patients requiring nonroutine discharge would be captured for a cost of two false positives per one true positive. In monetary terms, let us assume a hypothetical scenario in which each additional day in the hospital costs $\$ 1000$ and preoperative prediction results in elimination of 2 unnecessary postoperative hospital days. Furthermore, let us assume that obtaining insurance precertification requires 2 hours of case management time, valued at $\$ 100$ per hour. Overall, for every 3 patients predicted to have a nonroutine discharge by the model, 1 does have a nonroutine
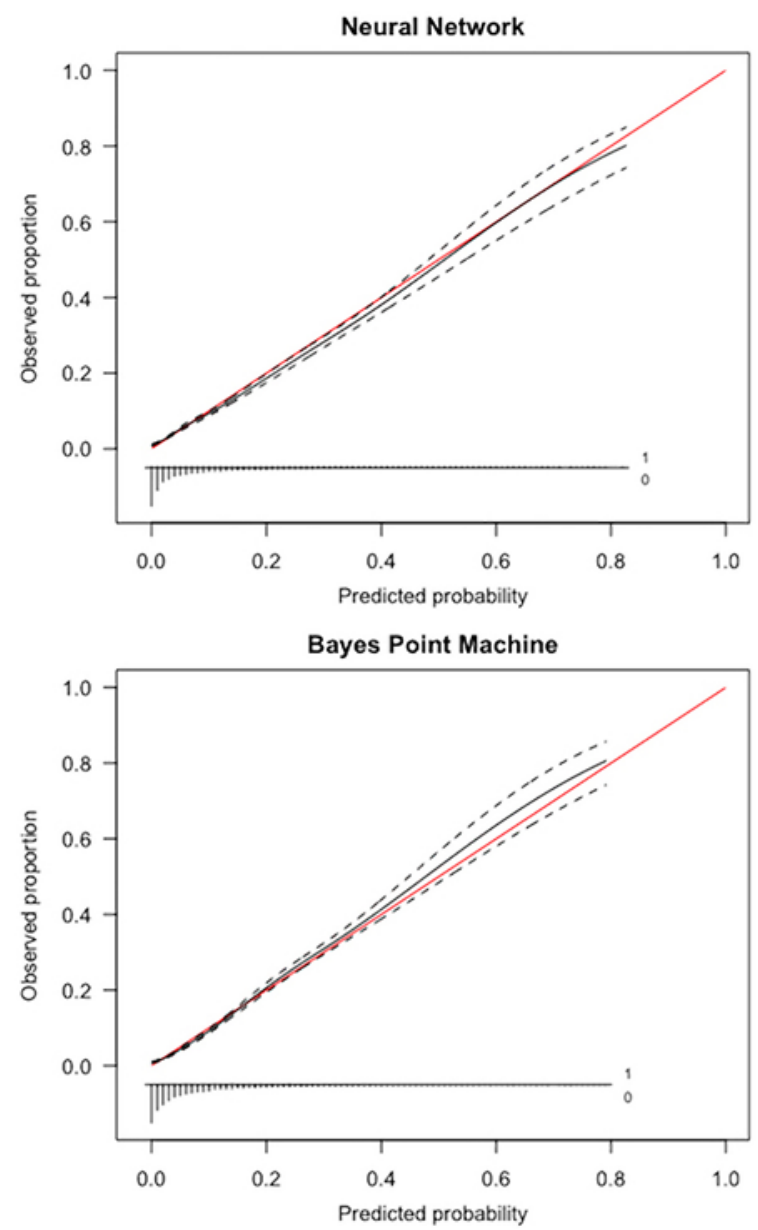

FIG. 2. Calibration of models on training set $(n=21,091)$. discharge and 2 do not. The cost saving is $\$ 2000$ for the patient with nonroutine discharge minus the $\$ 600$ for the 6 hours of total case management time ( 2 hours on the patient with nonroutine discharge plus 4 hours for the other 2 patients who did not have nonroutine discharge). The net cost saving is $\$ 1400$. Compared to the strategy of changing management for no patients, for all patients, or for patients based on the ASA class alone, the potential financial return of a targeted insurance precertification based on the neural network may offer significant cost savings to healthcare systems.

The limitations of this study include lack of psychosocial factors previously implicated in nursing home uti-
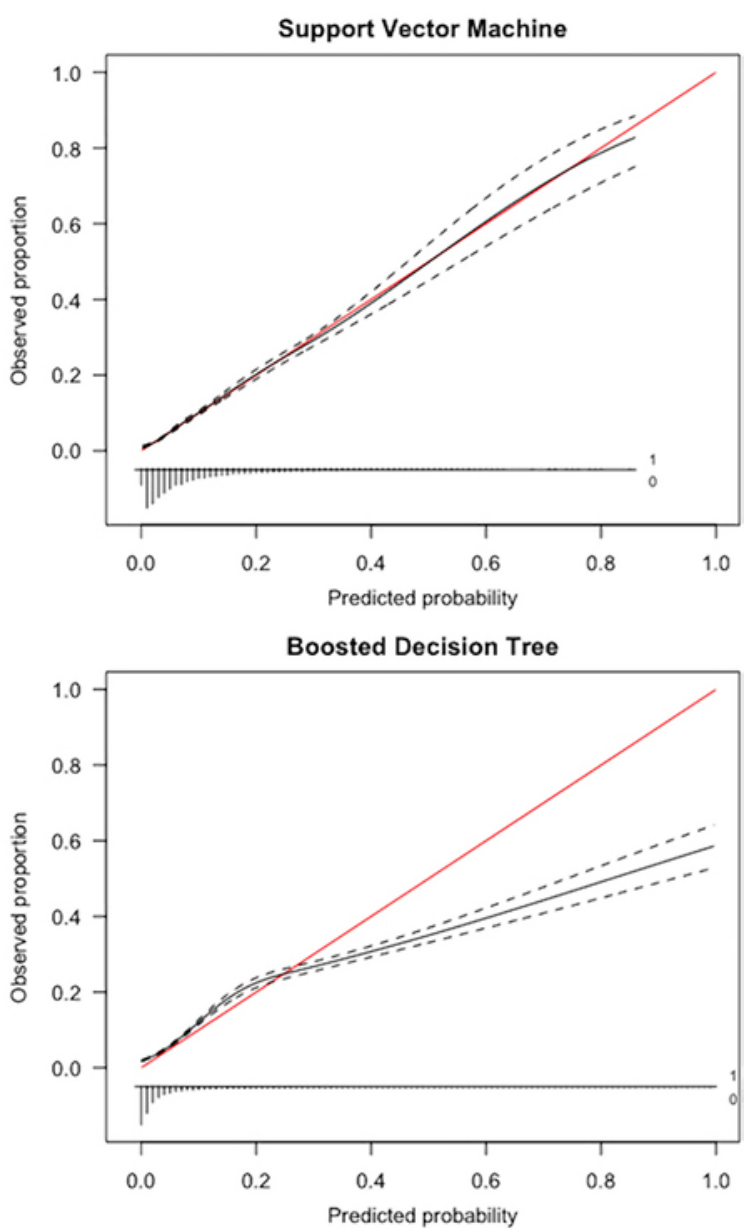
Karhade et al.

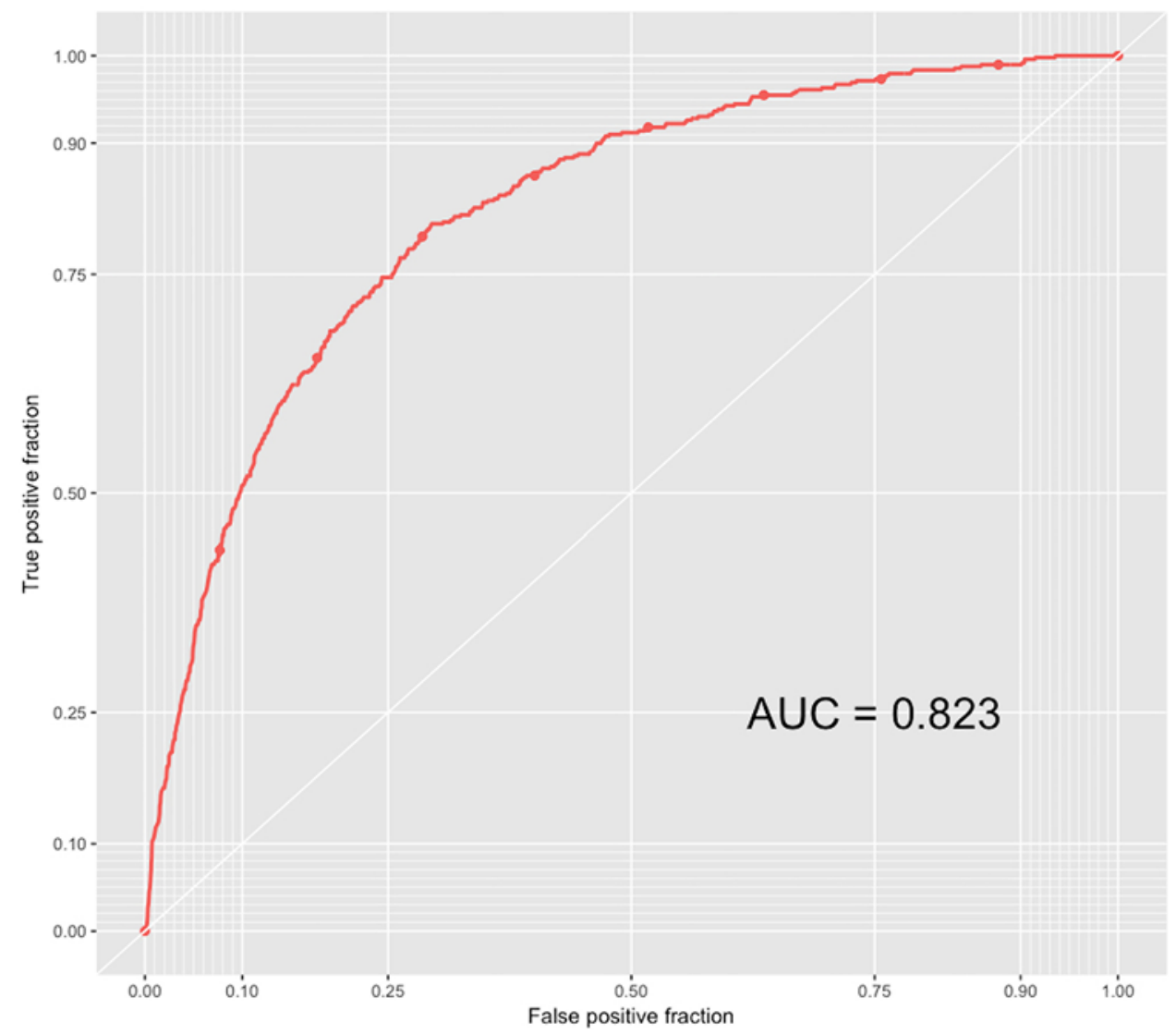

FIG. 3. Receiver operating curve for the neural network on testing set $(n=5273)$.

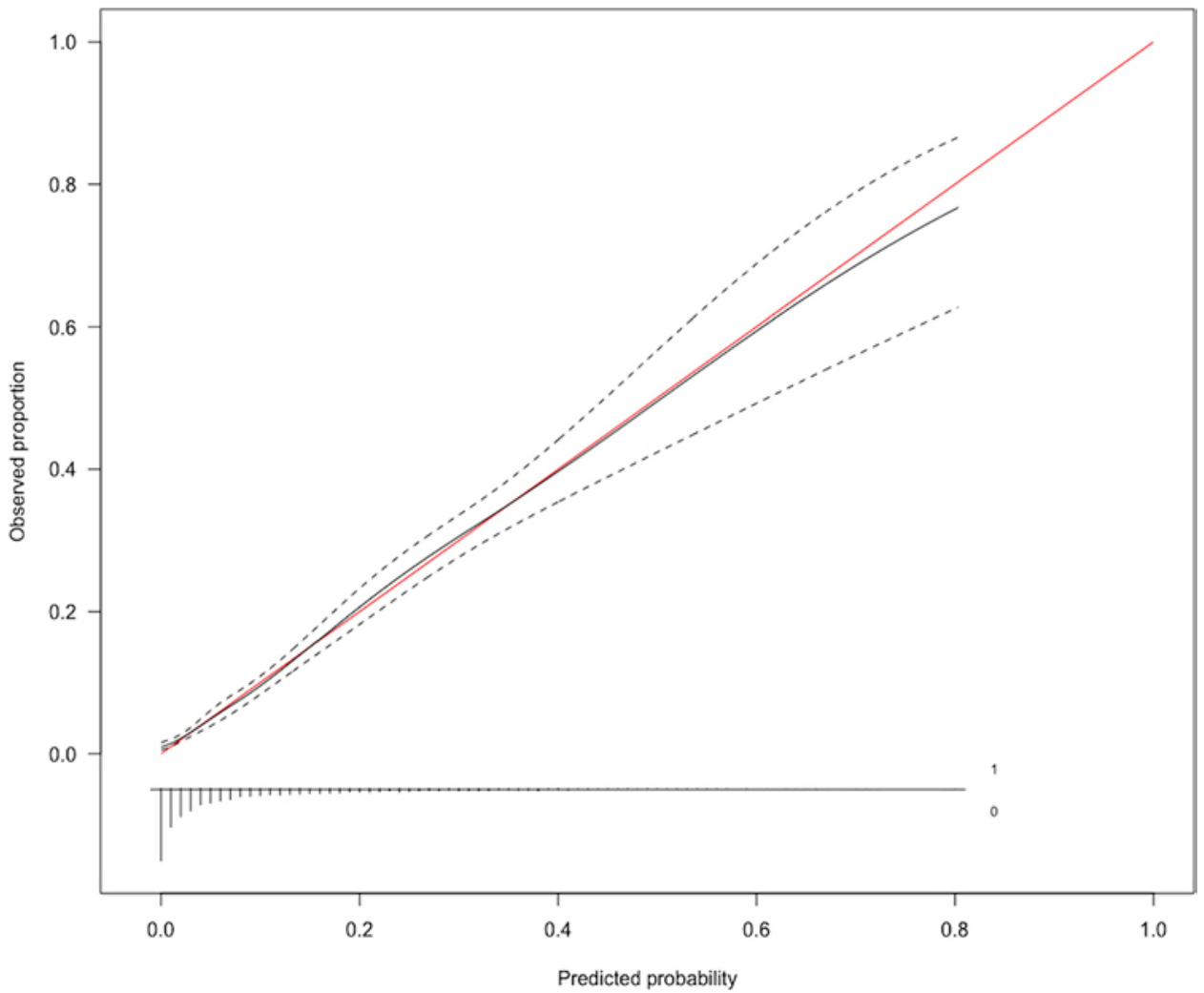

FIG. 4. Calibration plot for the neural network on testing set $(n=5273)$. 


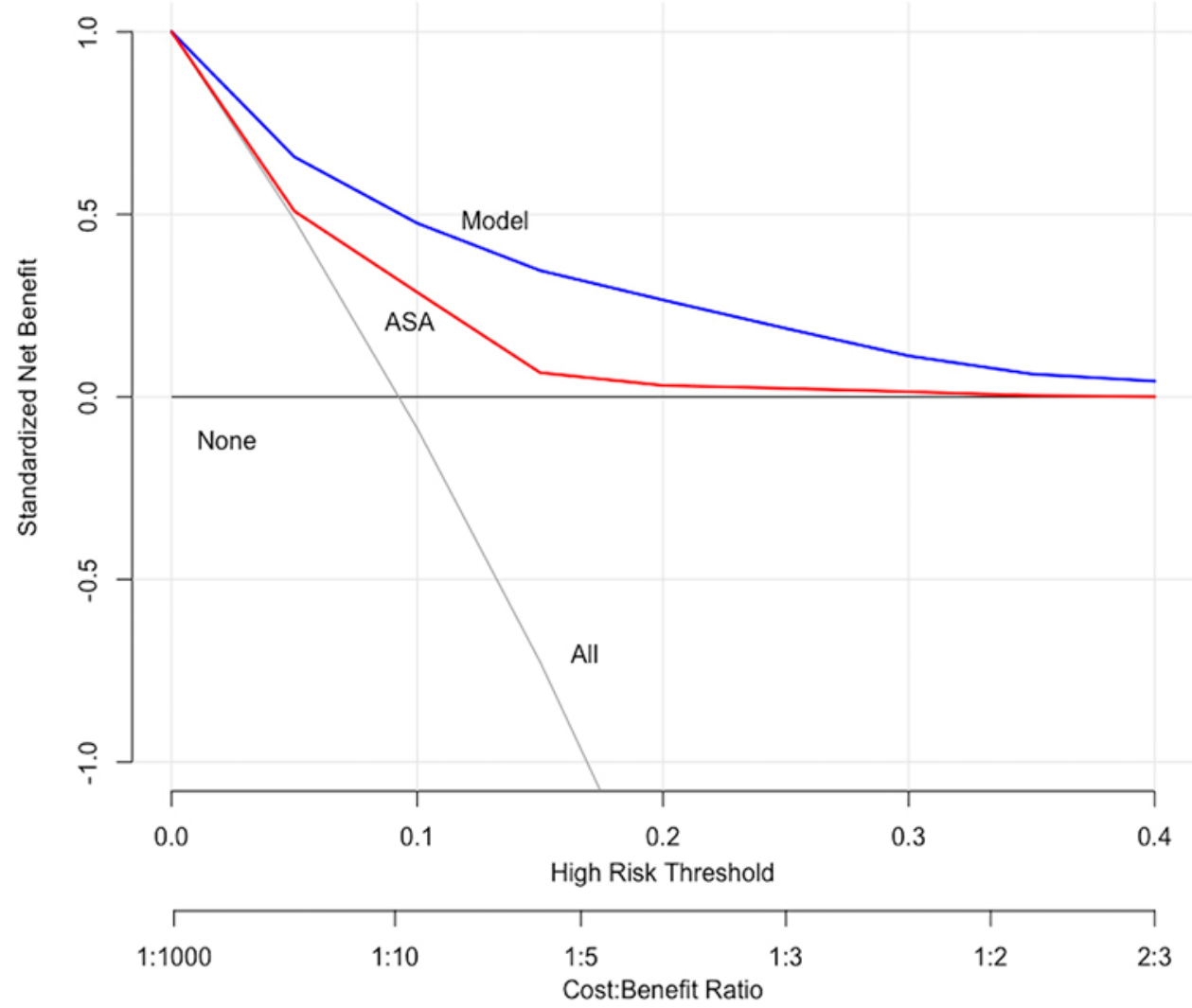

FIG. 5. Decision curve analysis for the neural network on testing set $(n=5273)$.

lization and nonhome discharge such as home residence with spouse or children, social support, depression, anxiety, and income. ${ }^{12,15}$ Additional important socioeconomic factors such as educational attainment, employment status, insurance status, insurance type, and median income of zip code of residence could not be evaluated in this study. Clinical factors such as duration of symptoms, preoperative neurological deficit, and previous spine surgery were unavailable in the NSQIP database. Furthermore, the quality of the NSQIP database may be less than that of single-institution prospectively collected, and physiciancurated data. In addition, although the neural network showed good performance on internal validation with a large multiinstitutional national sample, the external validation of this algorithm in an independent sample remains to be undertaken. External validation of this algorithm in physician-curated institutional databases and prospective studies is necessary for widespread use.

However, this study is still significant because it represents the first machine learning study for preoperative discharge planning in elective lumbar disc disease surgery. The blueprint for developing and assessing machine learning algorithms can be used in future studies that incorporate additional social factors previously studied for nonroutine discharge. Furthermore, no previous study in lumbar disc disease has deployed machine learning algorithms as an open-access decision tool; in the future, this model could be integrated into electronic health record systems as a digital decision support tool. Prior work with electronic health records for prediction of mortality and morbidity in surgery has shown promise for automated feature extraction and embedded machine learning. ${ }^{1}$ Integration of preoperative discharge planning into such a system for elective inpatient lumbar spine surgery is likely to offer significant benefit to healthcare systems seeking to enhance value-based care.

\section{Conclusions}

Machine learning algorithms show promising results on internal validation for the preoperative prediction of nonroutine discharges; external validation of these algorithms could help healthcare professionals substantiate the use of the open-access web application presented here for preoperative management in elective inpatient surgery for lumbar degenerative disc disorders.

\section{Acknowledgments}

The American College of Surgeons' NSQIP (ACS-NSQIP) and the hospitals participating in the ACS-NSQIP are the source of the data used herein; they have not verified and are not responsible for the statistical validity of the data analysis or the conclusions derived by the authors of this article.

\section{References}

1. Bihorac A, Ozrazgat-Baslanti T, Ebadi A, Motaei A, Madkour M, Pardalos PM, et al: MySurgeryRisk: development 
and validation of a machine-learning risk algorithm for major complications and death after surgery. Ann Surg [epub ahead of print], 2018

2. Bozic KJ, Ward L, Vail TP, Maze M: Bundled payments in total joint arthroplasty: targeting opportunities for quality improvement and cost reduction. Clin Orthop Relat Res 472:188-193, 2014

3. Collins GS, Reitsma JB, Altman DG, Moons KG: Transparent reporting of a multivariable prediction model for individual prognosis or diagnosis (TRIPOD): the TRIPOD Statement. BMC Med 13:1, 2015

4. Cutler DM, Ghosh K: The potential for cost savings through bundled episode payments. N Engl J Med 366:1075-1077, 2012

5. Deyo RA, Cherkin DC, Loeser JD, Bigos SJ, Ciol MA: Morbidity and mortality in association with operations on the lumbar spine. The influence of age, diagnosis, and procedure. J Bone Joint Surg Am 74:536-543, 1992

6. Di Capua J, Somani S, Kim JS, Lee NJ, Kothari P, Phan K, et al: Predictors for patient discharge destination after elective anterior cervical discectomy and fusion. Spine (Phila Pa 1976) 42:1538-1544, 2017

7. Di Capua J, Somani S, Lugo-Fagundo N, Kim JS, Phan K, Lee NJ, et al: Predictors for non-home patient discharge following elective adult spinal deformity surgery. Global Spine J 8:266-272, 2018

8. Fernández-Delgado M, Cernadas E, Barro S, Amorim D: Do we need hundreds of classifiers to solve real world classification problems. J Mach Learn Res 15:3133-3181, 2014

9. Hyder JA, Wakeam E, Habermann EB, Hess EP, Cima RR, Nguyen LL: Derivation and validation of a simple calculator to predict home discharge after surgery. J Am Coll Surg 218:226-236, 2014

10. Kanaan SF, Yeh HW, Waitman RL, Burton DC, Arnold PM, Sharma NK: Predicting discharge placement and health care needs after lumbar spine laminectomy. J Allied Health 43:88-97, 2014

11. Karhade AV, Larsen AMG, Cote DJ, Dubois HM, Smith TR: National databases for neurosurgical outcomes research: options, strengths, and limitations. Neurosurgery 83:333-344, 2018

12. Kersting RC: Impact of social support, diversity, and poverty on nursing home utilization in a nationally representative sample of older Americans. Soc Work Health Care 33:6787,2001

13. Kuhn M, Johnson K: Applied Predictive Modeling. Berlin: Springer, 2013, Vol 26

14. Luo W, Phung D, Tran T, Gupta S, Rana S, Karmakar C, et al: Guidelines for developing and reporting machine learning predictive models in biomedical research: a multidisciplinary view. J Med Internet Res 18:e323, 2016

15. Mancuso CA, Duculan R, Craig CM, Girardi FP: Psychosocial variables contribute to length of stay and discharge destination after lumbar surgery independent of demographic and clinical variables. Spine (Phila Pa 1976) 43:281-286, 2018

16. Maroco J, Silva D, Rodrigues A, Guerreiro M, Santana I, de Mendonça A: Data mining methods in the prediction of dementia: a real-data comparison of the accuracy, sensitivity and specificity of linear discriminant analysis, logistic regression, neural networks, support vector machines, classification trees and random forests. BMC Res Notes 4:299, 2011

17. McGirt MJ, Parker SL, Chotai S, Pfortmiller D, Sorenson JM, Foley K, et al: Predictors of extended length of stay, discharge to inpatient rehab, and hospital readmission following elective lumbar spine surgery: introduction of the CarolinaSemmes grading scale. J Neurosurg Spine 27:382-390, 2017

18. Miller DC, Gust C, Dimick JB, Birkmeyer N, Skinner J, Birkmeyer JD: Large variations in Medicare payments for surgery highlight savings potential from bundled payment programs. Health Aff (Millwood) 30:2107-2115, 2011

19. Murphy ME, Gilder H, Maloney PR, McCutcheon BA, Rinaldo L, Shepherd D, et al: Lumbar decompression in the elderly: increased age as a risk factor for complications and nonhome discharge. J Neurosurg Spine 26:353-362, 2017

20. Murphy ME, Maloney PR, McCutcheon BA, Rinaldo L, Shepherd D, Kerezoudis P, et al: Predictors of discharge to a nonhome facility in patients undergoing lumbar decompression without fusion for degenerative spine disease. Neurosurgery 81:638-649, 2017

21. Niedermeier S, Przybylowicz R, Virk SS, Stammen K, Eiferman DS, Khan SN: Predictors of discharge to an inpatient rehabilitation facility after a single-level posterior spinal fusion procedure. Eur Spine J 26:771-776, 2017

22. Passias PG, Poorman GW, Bortz CA, Qureshi R, Diebo BG, Paul JC, et al: Predictors of adverse discharge disposition in adult spinal deformity and associated costs. Spine J [epub ahead of print], 2018

23. Porter ME: A strategy for health care reform-toward a value-based system. N Engl J Med 361:109-112, 2009

24. Porter ME: What is value in health care? N Engl J Med 363:2477-2481, 2010

25. Raval MV, Pawlik TM: Practical guide to surgical data sets: National Surgical Quality Improvement Program (NSQIP) and Pediatric NSQIP. JAMA Surg 153:764-765, 2018

26. Schoenfeld AJ, Harris MB, Liu H, Birkmeyer JD: Variations in Medicare payments for episodes of spine surgery. Spine $\mathbf{J}$ 14:2793-2798, 2014

27. Slover J, Mullaly K, Karia R, Bendo J, Ursomanno P, Galloway A, et al: The use of the Risk Assessment and Prediction Tool in surgical patients in a bundled payment program. Int J Surg 38:119-122, 2017

28. Smith AL, Kulhari A, Wolfram JA, Furlan A: Impact of insurance precertification on discharge of stroke patients to acute rehabilitation or skilled nursing facility. J Stroke Cerebrovasc Dis 26:711-716, 2017

29. Ugiliweneza B, Kong M, Nosova K, Huang KT, Babu R, Lad SP, et al: Spinal surgery: variations in health care costs and implications for episode-based bundled payments. Spine (Phila Pa 1976) 39:1235-1242, 2014

\section{Disclosures}

Dr. Cha reports receiving received clinical or research support from the Gordon and Betty Moore Foundation and from the Donoghue Foundation. He is a consultant for Bio2, NuVasive, and $\mathrm{K} 2 \mathrm{M}$ and has received institutional fellowship support from NuVasive, K2M, and OMEGA.

\section{Author Contributions}

Conception and design: all authors. Acquisition of data: Schwab, Karhade, Thio. Analysis and interpretation of data: Schwab, Karhade, Ogink, Thio, Broekman. Drafting the article: Schwab, Karhade, Ogink, Broekman, Cha, Gormley, Hershman, Peul. Critically revising the article: Schwab, Ogink, Broekman, Cha, Gormley, Hershman, Peul, Bono. Reviewed submitted version of manuscript: Schwab, Cha, Gormley, Hershman, Peul, Bono. Statistical analysis: Schwab, Karhade, Ogink, Bono. Administrative/technical/material support: Schwab, Bono. Study supervision: Schwab.

\section{Correspondence}

Joseph Schwab: Massachusetts General Hospital, Boston, MA. jhschwab@mgh.harvard.edu. 